\title{
The effects of the GMF on the transition from Galactic to extragalactic cosmic rays
}

\author{
Alex Kääpä, ${ }^{a, *}$ Karl-Heinz Kampert $^{a}$ and Eric Mayotte ${ }^{a}$ \\ ${ }^{a}$ Bergische Universität Wuppertal, Gaußstraße 20, 42119 Wuppertal, Germany \\ E-mail: a.kaeaepae@uni-wuppertal.de, kampert@uni-wuppertal.de, \\ mayotte@uni-wuppertal.de
}

Understanding the nature of the transition from Galactic to extragalactic cosmic rays (GCRs and EGCRs) has become especially challenging in light of recent spectral and composition data. Galactic contributions appear to be disfavoured at energies beyond the "second knee" where the composition becomes lighter. At the same time, a dominant EGCR contribution at the "2nd knee" appears unlikely. As a result, the measured flux in the transition region cannot easily be accounted for. With the model-dependence of proposed extensions to both the Galactic and extragalactic contributions, a deeper understanding of CR propagation, particularly within the Galactic magnetic field (GMF), is in order. This is because propagation in this energy range shifts from diffusive to ballistic, which is expected to lead to a number of observable effects on CRs.

Using CRPropa3, we study these effects for rigidities between $10^{16}$ and $10^{20} \mathrm{~V}$. We identify various features at rigidities where the gyroradius is comparable to typical length scales of the Galaxy, suggesting causes related to changes in the propagation regime. We further quantify general modifications in the spectrum, composition and arrival direction of GCRs and EGCRs. We find that the GMF naturally induces a flux suppression of GCRs towards higher rigidities due to their increased leakage from the Galaxy. This, in consequence, would lead to an increase in the mean mass of GCR primaries up to energies around the "ankle" in the cosmic ray spectrum. It is also shown that the distribution of GCR arrival directions would be correlated with the Galactic plane for rigidities above $10^{17} \mathrm{~V}$ if sources are distributed evenly within the plane. EGCRs experience no flux modification in the GMF if injected isotropically. Injection of pure dipoles, as well as single source scenarios indicate that the GMF isotropises injected anisotropies below $10^{18} \mathrm{~V}$, but can still cause flux modifications which depend on the direction of the anisotropy.

$37^{\text {th }}$ International Cosmic Ray Conference (ICRC 2021)

July 12 th - 23rd, 2021

Online - Berlin, Germany

\footnotetext{
${ }^{*}$ Presenter
} 


\section{Introduction}

The nature of the transition from Galactic to extragalactic cosmic rays (GCRs and EGCRs, respectively) remains unresolved. Some past models appeared promising, as they could explain key features in the measured energy spectrum. These include the spectral softening at around $4 \cdot 10^{15} \mathrm{eV}$ (="knee") [1], a further softening at around $10^{17} \mathrm{eV}$ (=“second" or "iron knee") [2], a hardening at around $3 \cdot 10^{18} \mathrm{eV}$ (=“ankle") [3], and finally a high-energy cut-off beyond around $5 \cdot 10^{19} \mathrm{eV} \mathrm{[3].}$ The "knee" and "second knee" could be interpreted as the consequences of a rigidity-dependent ${ }^{1}$ end to the Galactic contribution, which would imply an increase in the mean mass of cosmic rays (CRs) beyond the "knee" [4]. Acceleration in the shock fronts of supernova remnants (SNRs), the prime candidate for the bulk of the GCR contribution [4] would naturally lead to spectral breaks at these energies, as most current simulations and models set their maximum acceleration rigidity at roughly $10^{15} \mathrm{~V}[4,5]$. The flattening at the "ankle" could be understood as the onset of the extragalactic contribution (see e.g. [6]). The so-called GZK effect [7, 8], where protons undergo photo-pion production in the cosmic microwave background (CMB) had further been proposed as the explanation the flux suppression at highest energies, provided that the composition is predominantly protonic. Heavier nuclei also interact with the CMB at highest energies, but in the form of photodisintegration [7-9]. A proton-dominated composition of EGCRs was favoured, however, as it would naturally describe the "ankle" as a consequence of electron-positron pair production of protons in the CMB, the so-called "proton-dip" models [10].

Recent spectral and composition data have complicated this picture. Due to the position of the "second knee" at $\approx 26$ times the energy of the "knee", both features were initially considered to arise from the same rigidity-dependent process, but for protons and iron nuclei, respectively. However, several challenges to this picture arise: On the one hand, composition data indicate that the maximum mean mass is reached at energies below the "second knee"2 [4]. On the other hand, according to most models, SNRs reach the maximum rigidity at around "knee" energies for protons. As heavier nuclei progressively reach the energy corresponding to this maximum rigidity, their fluxes sharply drop off. As a result, the total flux provided by SNRs is insufficient to account for the measured flux up to the "second knee" [4]. Lastly, a hardening between the "knee" and the "second knee" in the energy spectrum of the light component [11] suggests that different processes may partially be responsible for the two steepening features.

Most recent composition data at highest energies reveal a mixed composition of CRs, with a minimum mean mass at around the "ankle" and an increasing mean mass beyond that [12]. This disfavours the previously introduced "proton-dip" models where both the "ankle" and the high-energy cut-off result from interactions of protons with the CMB. A minimal model of EGCR production and propagation to describe the data above $5 \cdot 10^{18} \mathrm{eV}$, which considers all relevant interactions and energy loss processes, as well as an intrinsic maximum energy of the sources to test the nature of the high-energy cut-off, provides good agreement with composition and spectral data at these energies [13]. In it, the high-energy cut-off is predominantly an intrinsic feature of

\footnotetext{
${ }^{1}$ The rigidity $R$ is related to the energy of a CR particle via $E=R \cdot Z e$, where $Z e$ is the nuclear charge of the CR.

2 This could be explained with the onset of a light extragalactic component around this energy. However, a significant extragalactic contribution at these energies cannot be easily accounted for with the total available energy from structure formation in the early Universe and the conversion efficiency of this energy towards CR acceleration [4].
} 
the injection spectrum. However, this model does not attempt to describe the spectrum at energies below the "ankle", let alone the "second knee". Therefore, additional Galactic or extragalactic components have been introduced to describe the spectral and composition data in the transition region [4, 14]. These may be additional accelerators or accelerator classes [15-17], or interactions in the vicinity of EGCR sources that change spectral features [4, 18, 19].

Dipole anisotropy data further substantiates the energy ranges where the Galactic and extragalactic contributions dominate. Beyond the "knee" up to around $10^{16} \mathrm{eV}$, the phase of the measured dipole roughly aligns with the direction of the Galactic centre (GC) $[20,22] . \quad$ Beyond $\approx 10^{19} \mathrm{eV}$, however, the phase points distinctly away from it [23]. Between these two energies, no significant dipole has been measured, but the phases of the best-fit dipole anisotropies as measured by the Pierre Auger Observatory indicate a gradual shift from the GC towards the direction in which the extragalactic dipole points, especially beyond around $10^{18} \mathrm{eV}$ [21].

The main experimental features discussed above are sketched in figure 1. The top panel depicts the energy spectrum, with all aforementioned features visible. The second
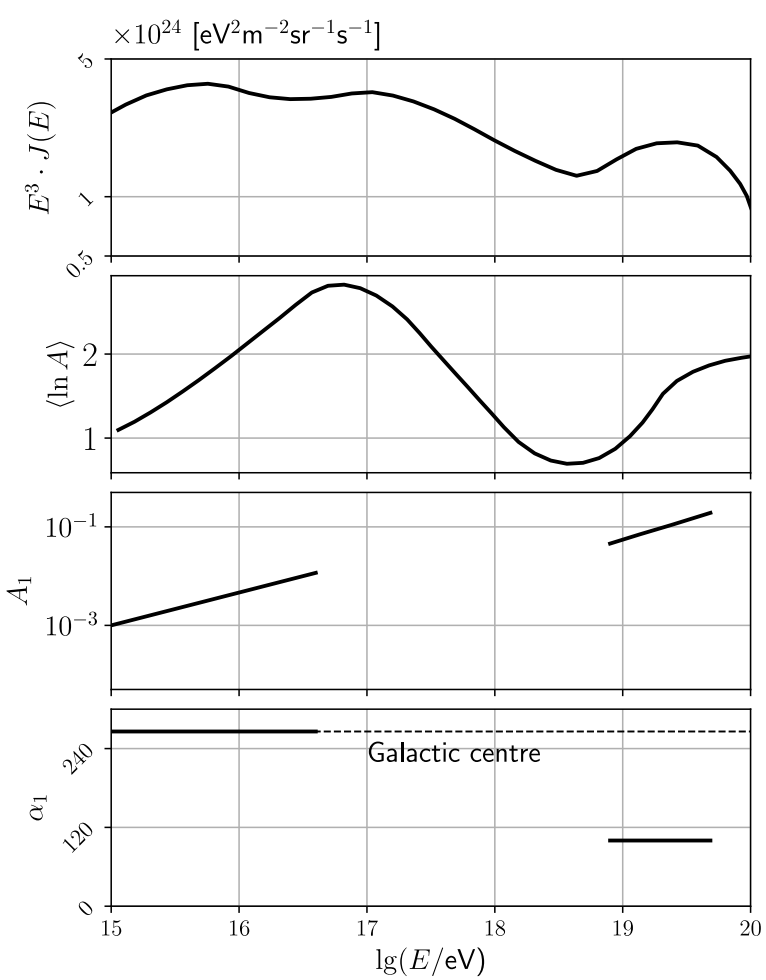

Figure 1: Summary of spectral, composition and dipole data via trend lines drawn to them, inspired by [1]. The spectrum and composition data are from [4], the dipole from [20, 21]. panel indicates the change in composition via the mean logarithm of the mass number $\langle\ln A\rangle$. The bottom two panels indicate the amplitude and phase of the large-scale dipole. They leave several open questions. More straightforward ones include: What is the nature of the "second knee"? Which types of accelerators could accelerate GCRs to energies beyond the "knee" and provide a sufficient flux? What explains the decrease in mean mass towards the "ankle"? What is the minimum energy at which EGCRs contribute significantly?

In answering these questions, the effect of the Galactic magnetic field (GMF) on both the flux and composition of $\mathrm{CRs}^{3}$ has not been fully considered. It has already been shown using simulations with two GMF models that the propagation of CRs on average becomes ballistic at a rigidity of $\approx 6 \mathrm{EV}$ [24]. Generally, the gyroradius ${ }^{4}$ provides a good intuitive understanding of this shift in propagation regimes. Fig. 2 depicts the gyroradius $r_{g}$ as a function of rigidity $R$ for

\footnotetext{
${ }^{3}$ Studies of the effect of the GMF on the arrival direction distribution have been performed upper ends of this energy range, where EGCRs are expected to dominate [24-26].

${ }^{4}$ The gyroradius $r_{g}$ provides the strength of deflection of particle with rigidity $R$ in a magnetic field of strength $B$ via $r_{g} \approx 11 \frac{R[\mathrm{PV}] \cdot \beta_{\perp}}{B[\mu \mathrm{G}]} \mathrm{pc}$, where $\beta_{\perp}=\frac{v_{\perp}}{c}$.
} 
typical magnetic field strengths of the Galaxy. Horizontal lines are added to indicate two important Galactic length scales, the width and radius of the Galactic plane (GP), $h_{\mathrm{GP}}\left(\approx \mathrm{a}\right.$ few kpc) and $r_{\mathrm{GP}}$ $(\approx 10-20 \mathrm{kpc})$, respectively, but also the approximate coherence length $\lambda(\approx 100 \mathrm{pc})$ of the random component of the GMF [27].

For typical field strengths of the GMF $(\approx$ a few $\mu \mathrm{G})$, the gyroradius is $r_{g} \approx$ a few $\cdot R[\mathrm{EV}] \mathrm{kpc}$. At $R=6 \mathrm{EV}, r_{g}$ approaches $r_{\mathrm{GP}}$, and $\mathrm{CR}$ propagation can be characterised as ballistic. At rigidities where $r_{g}<\lambda$, but particularly at those corresponding to the "knee" for protons (where $\left.r_{g}<0.001 \cdot r_{\mathrm{GP}}\right)$, CRs are effectively confined in the GP, and propagate diffusively. In the transition region from GCRs to EGCRs, the propagation changes from diffusive to ballistic. This may plausibly affect the flux of both GCRs and EGCRs. GCRs in-

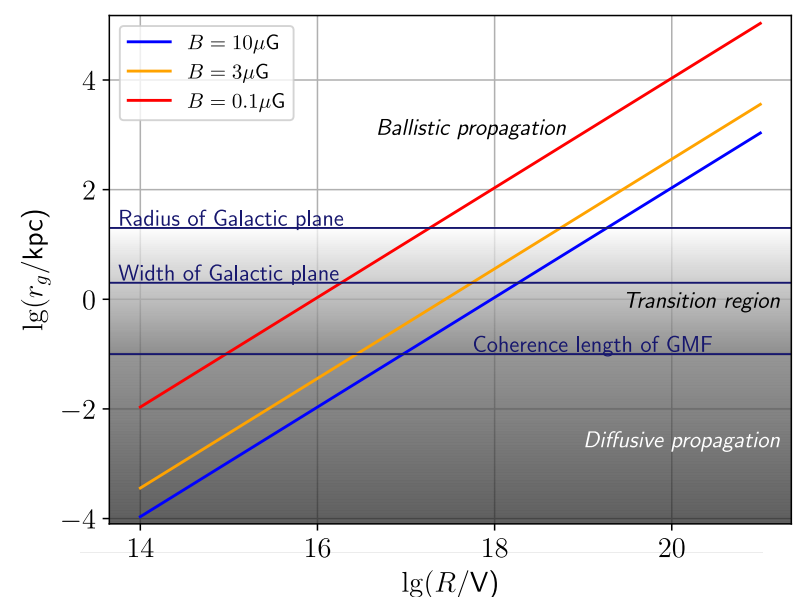

Figure 2: Gyroradius $r_{g}$ as a function of rigidity $R$ for typical Galactic magnetic field strengths. Horizontal lines signify typical Galactic length scales. creasingly leak out of the Galaxy with increasing rigidity ${ }^{5}$. EGCRs are increasingly shielded from the Galaxy with decreasing rigidity, while those that do reach the GP are confined therein.

To investigate the propagation effects of GCRs and EGCRs in the GMF, we carry out MonteCarlo-based simulations of CR propagation. We perform forward-tracking studies, as backward tracking is not viable in the context of GCRs ${ }^{6}$. Forward tracking also allows for investigating the change of EGCR densities in the Galaxy. We propagate only protons as we are studying only deflections in the GMF and neglecting interactions, i.e. all effects are merely rigidity-dependent ${ }^{7}$. Later on, anisotropic injections of EGCRs via the Galactic lensing scheme will be studied. To create the Galactic lens, backward tracking simulations of anti-protons are performed. The details of the injected anisotropies as well as the creation and testing of the lens are given in section 4.2.

\section{Simulation setup}

The simulation studies are performed with the help of the Monte-Carlo-based cosmic-ray propagation software CRPropa3 [28]. We employ the ballistic propagation module where individual particles are tracked solving the equation of motion using the Cash-Karp algorithm. In each simulation, a minimum and maximum step size, $d_{\min }$ and $d_{\max }$, can be chosen. The minimum gyroradius (i.e., where $\beta_{\perp}=c$ ) of a PV-rigidity particle in a $>10 \mu \mathrm{G}$-field is $r_{g}<1 \mathrm{pc}$, setting a safe minimum step size to $d_{\min }<0.1 \mathrm{pc}$. We conservatively opted for $d_{\min }=0.01 \mathrm{pc}$. The JF12 model [27] is used

\footnotetext{
${ }^{5}$ Increasing leakage of CRs from their host galaxies with rigidity would provide a measure of the expected EGCR flux; this will not be further investigated in this work, though.

${ }^{6}$ In such studies, (anti-)particles are tracked from the observer to the source. In the case of GCRs, the GP is the observer, which is crossed several times during propagation. Therefore, backward-tracking studies do not allow for the unique identification of the sources.

${ }^{7}$ Results for other nuclear species can be retrieved via appropriate scaling with the corresponding charge number.
} 
as the GMF model, since it is currently most commonly used. All three components, the regular, striated (large-scale random) and (small-scale) random component, are included. The random seed which sets the latter two components is rethrown for each particle. Two kill conditions for the simulation are set. The first is the edge of the Galaxy (i.e. the edge of the GMF), which is a $20 \mathrm{kpc}$ shell (henceforth called the Galactic shell) around the GC for the JF12 model. The second condition is a maximum trajectory length of $1 \mathrm{Gpc}$.

The sources and observers are defined as follows: The source of GCRs is a disk centered on the $\mathrm{GC}$ with a radius of $20 \mathrm{kpc}$. It has a uniform source density and from each point CRs are injected isotropically. The source of EGCRs also mimics that of isotropic injection via a uniform source density along the Galactic shell with a Lambertian injection direction distribution. There are two types of observers. The first one is the GP itself, parameterised via a cylinder of $100 \mathrm{pc}$ thickness centered around the GC. In the case of GCRs, the radius is set to $20 \mathrm{kpc}$, same as their source. For EGCRs, the radius was reduced to $19.5 \mathrm{kpc}$. This is motivated by the fact that the drop-off of the strength of the GMF at the edge of the Galaxy is discontinuous for the JF12 field, allowing low-rigidity particles to penetrate further into the Galaxy than they would in the case of a continuous drop-off of the field strength beyond the edge of the Galaxy. For EGCRs, this introduces a source of systematic bias if the GP observer reached the edge of the Galaxy. The surplus particles are eventually reflected back outside the Galaxy. Therefore, reducing the radius functions as a fiducial cut to recover the behaviour which would occur for a continuous drop-off of the GMF. The second observer is Earth, and is parameterised as a shell centered at the location of Earth in Galactic coordinates $(x=-8.5 \mathrm{kpc})$. The shell has a variable radius from $5 \mathrm{pc}$ to $1 \mathrm{kpc}$. With the variable radius, we seek to identify artifacts stemming from the finite observer shell size.

The rigidity range of $10^{16}-10^{20} \mathrm{~V}$ is set for the simulated particles ${ }^{8}$. Ideally, the entire rigidity range of the transition region would be covered. Below $10^{16} \mathrm{~V}$, however, simulation of a large number of particles becomes computationally unfeasible with the available resources ${ }^{9}$. Finally, all CRs are injected with an $R^{-1}$-spectrum, to make possible modifications in the spectrum visually discernible.

\section{Propagation effects}

In the following, we describe and quantify the propagation effects as a function of the rigidity of CRs. Given the fact that GCRs will only be considered until they escape from the Galaxy while EGCRs experience a shielding effect before entering the GMF for further propagation, we discuss GCRs and EGCRs separately.

\footnotetext{
${ }^{8}$ In the study of the large-scale propagation effects in 3, we extend the rigidity range downward by an order of magnitude to $10^{15}-10^{20} \mathrm{~V}$ as the relatively large observer size (the GP) allowed for a sufficiently small number of simulated particles.

${ }^{9}$ At such energies, simulations of diffusive propagation solving the transport equation become more desirable. Such a diffusion module is included in CRPropa3, but was not employed for this work. Generally, a cross-check between the two modules across the rigidity range, where the change in propagation regimes from diffusive to ballistic occurs, is of interest.
} 


\subsection{GCRs - Confinement}

GCRs make up a bulk of the low-energy flux in the energy spectrum in the transition region and thus also of the low-rigidity part of the investigated rigidity range. At rigidities of $10^{16} \mathrm{~V}$, the corresponding gyroradius of CRs created in the GP is on the order of $10 \mathrm{pc}<0.001 \cdot r_{\mathrm{GP}}$. Therefore, GCRs are expected to propagate diffusively and be strongly confined in the GP. With a strong confinement in the GMF (i.e. large residence times in the GP), GCRs have an increased probability of reaching any point in the GP (including the position of the Earth observer). As rigidity increases, the confinement decreases and the probability of reaching any point in the GP along with it. Thus, the flux at Earth is expected to decrease. When the rigidity increases to a level where the gyroradius approaches the characteristic length scales of the GP, the confinement of GCRs in the GP disappears. The expected flux approaches that from the magnetic-field-free case and returns to the injected spectral index. The arrival direction is expected to be largely isotropic for low rigidities and to increasingly point towards the direction of the source (i.e. the GP) as rigidity increases.

\subsection{EGCRs - Confinement and shielding}

EGCRs dominate the high-energy flux of the energy spectrum in the transition region. Originating from outside the Galaxy, they have to propagate a larger distance through the GMF before reaching the GP. At high rigidities, the fraction of EGCRs that reach a point in the Galaxy (including the GP) can be inferred from the field-free case. As the rigidity falls below the characteristic length scales of the GP, the effect of the GMF starts to play a role. With the field strength significantly increasing towards the GP, the GMF increasingly shields EGCRs from the GP. At the same time, the ones that do reach the GP increasingly experience the confinement discussed above for GCRs. Therefore, we have two counteracting effects for EGCRs: shielding from and confinement in the GP, both of which increase as rigidity goes down. Whether one of them dominates or both cancel each other out remains to be investigated. Given the structure of the GMF, its effect on the arrival direction of EGCRs at Earth is less simple to predict. When injected isotropically, the arrival direction is expected to remain isotropic for all rigidities [29]. For an anisotropic injection of EGCRs, the degree of smearing and isotropisation of EGCRs in the GMF towards lower rigidities remains to be studied. In addition, the injection directions for which the Galaxy is magnetically more (less) transparent or opaque from the vantage point of Earth, will shift with rigidity [25], so that additional spectral modifications may occur.

\subsection{Quantification of propagation effects}

To parameterise the confinement in the GP, the relative residence time, $t_{\text {rel }}=t_{\text {in }} / t_{\text {tot }}$, of CRs in the GP is considered. Here, the residence time of each CR within the GP, $t_{\text {in }}$, is normalised to its total residence time, $t_{\text {tot }}$, in the Galaxy. As confinement increases, $t_{\text {rel }}$ is expected to increase and vice versa. The median value of $t_{\text {rel }}$ as a function of rigidity is plotted in Fig. 3a for both GCRs and EGCRs. Both follow trends as expected: At low rigidities, confinement is high with CRs spending the majority of their total time in the Galaxy within the GP. Above around 0.1 EV, $t_{\text {rel }}$ decreases rapidly and above a few EV, it flattens out, as the corresponding gyroradius exceeds 


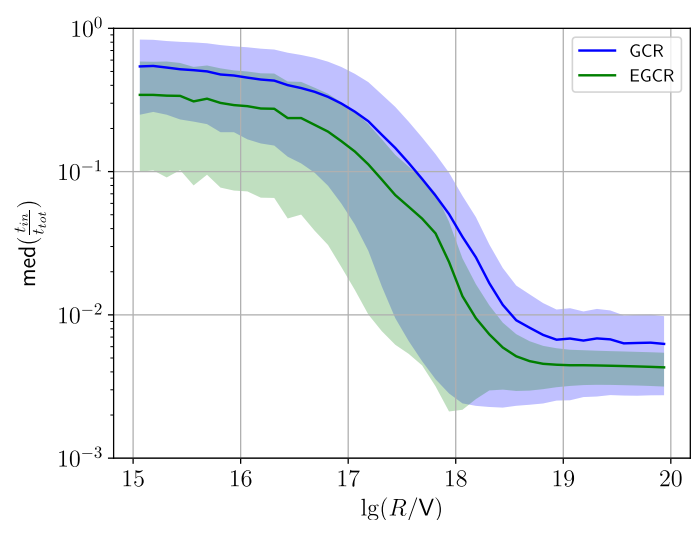

(a) Median (lines) and the median absolute distance (shaded areas) of the relative residence times of GCRs (blue) and EGCRs (green) as a function of rigidity.

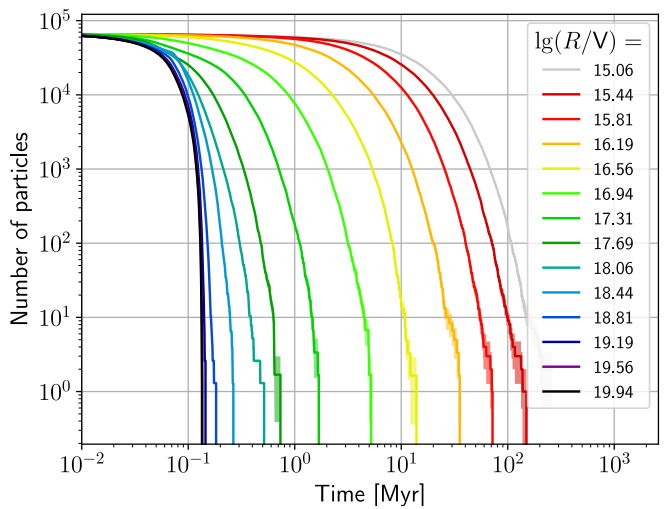

(b) Evolution of the number of GCRs in the Galactic volume with time for various rigidities signified in the legend.

Figure 3: Quantification of the confinement of GCRs and EGCRs.

the characteristic length scales of the Galaxy. The decreased confinement or increased leakage of GCRs with rigidity is a central aspect of the transition region and deserves further elucidation. For this purpose, we calculate the number of GCRs propagating within the Galactic volume as a function of time for various rigidities, as depicted in Fig. 3b. The plot clearly shows a decreasing confinement, or increasing leakage, of CRs as rigidity increases. In addition, the plot provides an estimate of the residence times ${ }^{10}$ of GCRs, which approach values of about $20 \mathrm{Myr}$ at lowest rigidities [30], supporting the approach utilised, despite being somewhat at the higher end ${ }^{11}$.

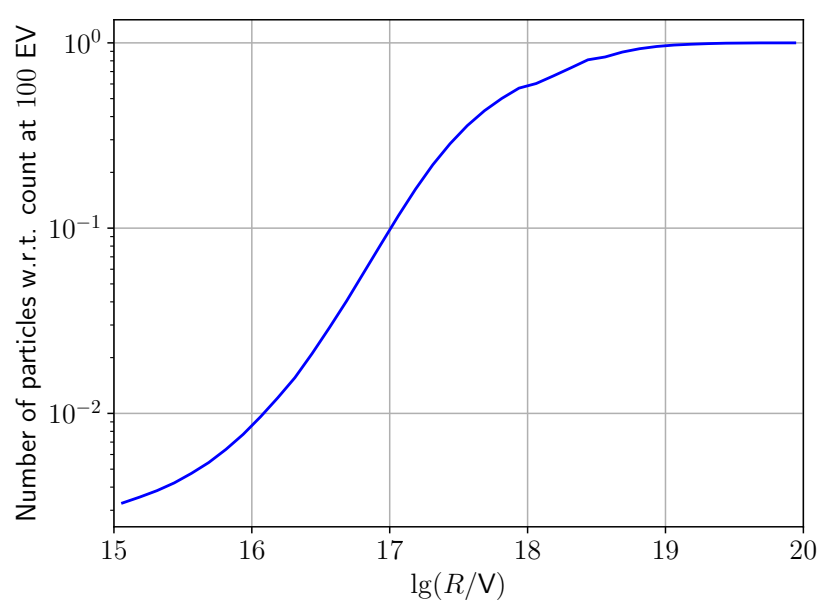

Figure 4: The total number of EGCRs reaching the GP as a function of rigidity.

The shielding of EGCRs from the GP is parameterised via the CR count reaching the GP, $N_{\mathrm{CR}, \mathrm{GP}}$, which is depicted in Fig. 4 as a function of rigidity. Again, we see the expected trend with rigidity. At high rigidities, $N_{\mathrm{CR}, \mathrm{GP}}$ saturates. Below a few $\mathrm{EV}$, when the gyroradius becomes smaller than the radius of the Galaxy, the count starts to decrease. The trend becomes steepest at around $10^{17} \mathrm{~V}$, below which it slowly flattens out as the gyroradius becomes so small with respect to the radius of the Galaxy $\left(\frac{r_{g}}{r_{\mathrm{GP}}} \rightarrow 0\right)$ that propagation becomes entirely diffusive.

\footnotetext{
${ }^{10}$ We roughly estimated the residence time as the time after which the CR count has fallen to $\frac{1}{e}$ of its initial value.

${ }^{11}$ Indeed, it is believed that the JF12 model overestimates the strength of the random component [25].
} 


\section{Effects on flux, composition and arrival direction}

With the large-scale propagation effects (confinement and shielding) identified and associated with the strength and large-scale structure of the GMF, we will now study how these effects manifest for observables such as flux, composition, and arrival directions. For this purpose, GCRs and EGCRs are forward tracked from their respective sources to Earth across the entire simulated rigidity range.

For the case of GCRs, the reduced confinement in the GP is expected to lead to a flux suppression towards higher rigidities as the probability of reaching any single point in the GP decreases. Such a rigidity-dependent flux suppression, in turn, leads to an increasing mean mass of a mixed composition of CRs with energy up until a few $\mathrm{EeV}\left(\approx E_{\text {ankle }}\right)$. This is where the spectrum for protons flattens and their contribution to the all-particle flux increases. Also, the arrival direction distribution is expected to increasingly align with the GP.

For EGCRs, Liouville's theorem predicts a flux conservation and an isotropic arrival direction in case of isotropic injection [29]. This implies, however, confinement and shielding exactly cancel. This is worthy of verification in its own right. Furthermore, confirming that Liouville's theorem does, in fact, hold, further substantiates the proposed simulation approach and the validity of the subsequent backward-tracking studies. Similarly, conservation of isotropy rules out any intrinsic anisotropy that the GMF may introduce. This we also seek to confirm. In case of an anisotropic injection, the effects of propagation in the GMF are less easy to predict. Due to the shifting position in the sky in the degree of transparency of the GMF, flux modifications may occur depending on the nature of the anisotropy. In terms of the arrival direction, lower-rigdity particles are expected to be increasingly deflected, and the injection direction distribution is increasingly smeared out. The injection of anisotropy, therefore, also serves to test the degree of isotropisation by the GMF.

\subsection{GCRs}

To measure the flux and the rigidity spectrum of GCRs arriving at Earth, the CR count crossing the shell of various radii centered on Earth is measured. The count is then scaled to the expected count from the field-free case. In this way, the flux suppression towards higher rigidities appears more like a flux enhancement towards lower rigidities. We found an increase in this flux enhancement with decreasing observer shell radius with no indication of convergence. However, the smallest shell radius analysed, $r_{\mathrm{obs}}=5 \mathrm{pc}$, is smaller than the gyroradius $r_{\mathrm{g}} \approx 10 \mathrm{pc}$ at lowest rigidities. Within the shell, propagation therefore is ballistic, and a significantly larger flux enhancement is not expected. In Fig. 5a, the measured rigidity spectrum is depicted for $r_{\mathrm{obs}}=5 \mathrm{pc}$ (blue line). With the expectation of a flattening of this suppression towards even lower rigidities, we fitted a sigmoid function to the spectrum in the form $f(x)=A /(1+\exp ((x-C) / B))+D$ (where $A, B, C$ and $D$ are free parameters ${ }^{12}$; see red line). This was primarily done to allow for the analysis of the composition for a broader energy range ${ }^{13}$. While the validity of the extrapolation to lower rigidities

12 The parameter $A$ gives the initial flux before the confinement begins to decrease. $B$ and $C$ define the steepness and the shift (along the $x$-axis) of the fitting function; they were introduced to retrieve the same steepness of the rigidity spectrum beyond $\approx 10^{17.5} \mathrm{~V}$ for all shell sizes, that was observed. $D$ gives the final flux in the field-free case.

${ }^{13}$ For the simulated rigidity range, the mixed-mass energy spectrum would only be defined between $10^{16+\lg 26}=$ $10^{17.4} \mathrm{eV}$ and $10^{20} \mathrm{eV}$, i.e. at energies where the Galactic contribution to the measured energy spectrum is increasingly negligible. 


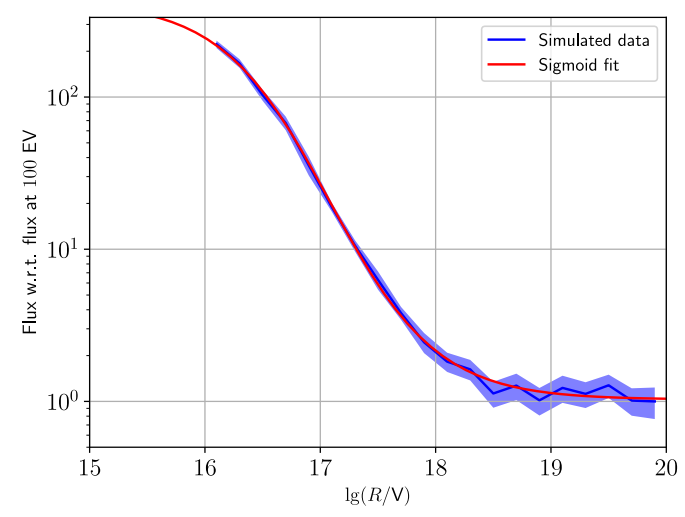

(a) The measured (blue line) modification of the rigidity spectrum of GCRs due to propagation effects in the GMF with $r_{\mathrm{obs}}=5 \mathrm{pc}$ together with a sigmoid fit (red line).

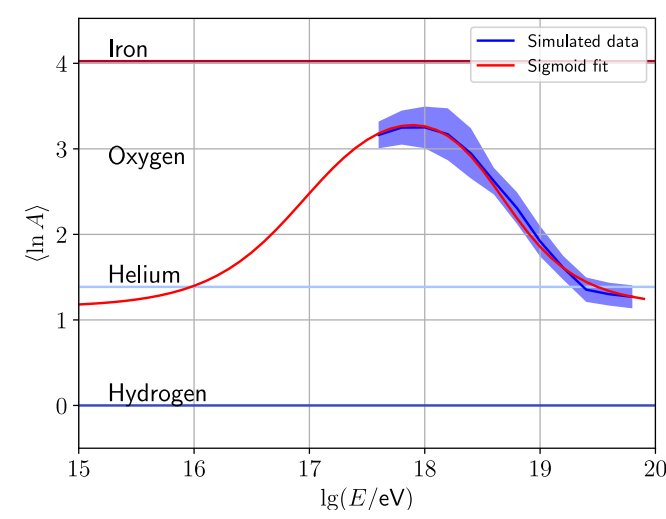

(b) The measured (blue line) modification of $\langle\ln A\rangle$ of GCRs due to propagation effects in the GMF as a function of rigidity together with a sigmoid fit (red line).

Figure 5: Effects of propagation on the the flux and composition of GCRs.

is rather uncertain, this does allow for a qualitative understanding of the expected trend of the GCR flux.

The composition of CRs is quantified via the mean logarithm of the mass number $\langle\ln A\rangle$. This quantity is calculated via the sum of the energy-dependent fractions of the flux $f_{i}(E)$ of each nuclear species $i$, multiplied by the logarithm of their respective mass numbers $\ln A_{i}:\langle\ln A\rangle=\sum_{i} f_{i}(E)$. $\ln A_{i}$. The fractions $f_{i}(E)$ are determined by multiplying their energy spectra $\phi_{i}(E)$ to their initial fraction $f_{i}^{0}$, normed via the total flux: $f_{i}(E)=\frac{\phi_{i}(E) \cdot f_{i}^{0}}{\sum_{i} \phi_{i}(E) \cdot f_{i}{ }^{0}}$. The individual spectra, in turn, follow from the measured rigidity spectrum shown in Fig. 5a: $\phi_{i}(E)=\phi\left(R=\frac{E}{Z_{i} \cdot e}\right)$. We injected a fourcomponent composition $(i=\mathrm{H}, \mathrm{He}, \mathrm{O}, \mathrm{Fe})$ with $\left[f_{\mathrm{p}}^{0}, f_{\mathrm{He}}^{0}, f_{\mathrm{O}}^{0}, f_{\mathrm{Fe}}^{0}\right]=[45.0 \%, 35.8 \%, 9.6 \%, 9.6 \%]$ (adapted from [18]).

The resulting modification of $\langle\ln A\rangle$ due to propagation in the GMF as a function of energy is depicted in Fig. 5b. Indeed, we find an increasing mean mass of GCRs all the way up to roughly "ankle" energies, beyond which it decreases again. However, we wish to emphasise that this peak is solely an effect CR leakage from the Galaxy. For a quantitative comparison to data we would need to add a flux of EGCRs and introduce a riditidy-dependent cut-off to GCR
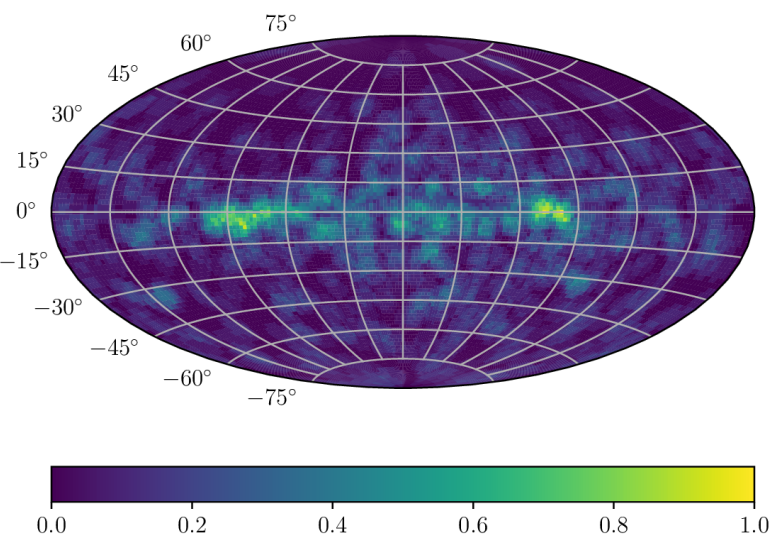

Figure 6: Smoothed sky map ( $5^{\circ}$ opening angle) of the arrival direction distribution of GCRs above $10^{17} \mathrm{~V}$. sources, both of which would shift the position of the peak (i.e. maximum mean mass) towards lower energies. This will be subject of further studies.

The rigidity beyond which the arrival direction distribution is found to correlate strongly with 
the direction of the GP is roughly $10^{17} \mathrm{~V}$. The skymap of the arrival direction distribution for all GCRs with a rigidity higher than this value is depicted in Fig. 6.

\subsection{EGCRs}

As for GCRs, the flux and rigidity spectrum of EGCRs are calculated via the CR count crossing the shell paramterising Earth scaled to the count expected in the field-free case. In Fig. 7, the rigidity spectrum for various shell radii is depicted. For larger sphere radii, there appears to be a flux suppression towards smaller rigidities. This suppression appears to vanish, however, as the observer approaches a point (i.e. $r_{\mathrm{obs}} \rightarrow 0$ ). In fact, shielding and confinement cancel exactly for isotropically injected EGCRs. Due to this flux conservation, the composition also remains the same after propagation in the GMF. We find an isotropic arrival direction distribution at Earth across the entire rigidity range, meaning that the GMF does not introduce any intrinsic anisotropy.

Having confirmed the conservation of flux and isotropy for isotropically injected EGCRs, the effects on anisotropically injected EGCRs remain to be investigated. For this purpose, the lensing method $[24,31]$ is particularly suitable. The lensing approach is a popularly employed scheme in calculating the effects of deflection of EGCRs in the GMF. It utilises the fact that the extragalactic sources are situated significantly further away than the extent of the Galaxy, due to which the latter can be viewed as a point. In such a case, the position at which EGCRs penetrate

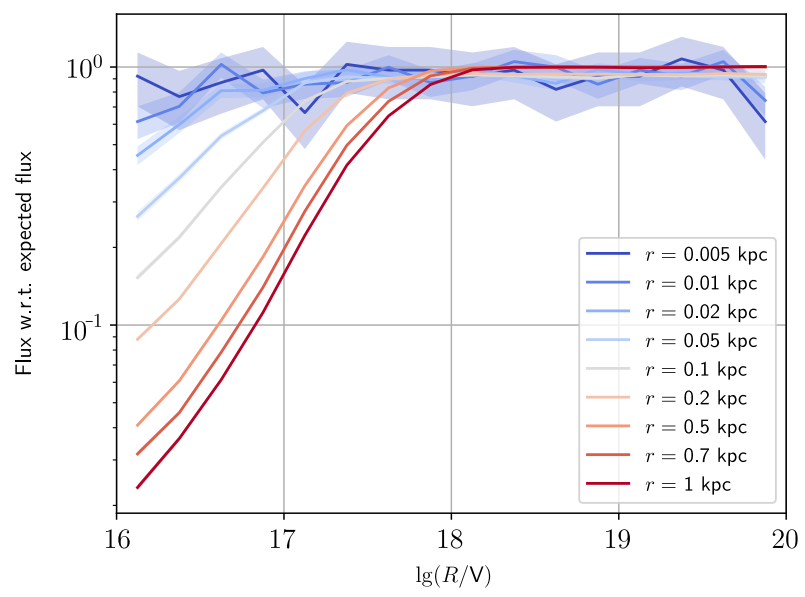

Figure 7: The measured rigidity spectrum of EGCRs for various observer shell radii. the Galaxy can be ignored and we are left with only two variables that quantify the deflection in the GMF, the injection and arrival direction. By tracking several particles that cross the Galactic shell and the Earth observer and binning the injection and arrival directions, a matrix associating a set of arrival directions to each injection direction can be defined. This matrix normed via the one-norm (i.e. number of simulated particles) yields the lens with which the arrival direction distribution for any given injection direction distribution can be calculated. Calculating such a lens for several rigidity bins allows for the analysis of spectral modifications. For this, each rigidity-dependent matrix has to be normed via the maximum one-norm of the entire set of matrices. In case of a flat distribution with rigidity, the one-norm is the same for each sub-lens. Given that the GMF conserved flux and isotropy for isotropically injected EGCRs across the entire simulated rigidity range, building the lens via backward tracking of anti-particles and a subsequent inversion of the directions becomes an option. This is only valid if time reversibility can be assumed. For sub-"ankle" energies, this is not clear, as deflections are very strong and could effectively function as collisions. To test this, we compared the injection direction distributions of forward-tracked protons that arrive isotropically at Earth with the inverted distribution of directions with which anti-protons injected isotropically at Earth leave the Galaxy. We found good agreement between the two distributions. 
To test the lens, we confirmed that the lens yields an isotropic arrival direction distribution and a conserved flux in the case of isotropic injection.

With a reasonable confidence in the functionality of the lens, two different (pure) anisotropy scenarios are tested. The first is the largest-scale anisotropy that may be measured, namely that of a dipole. The second is that from a point source, the strongest type of anisotropy that may be injected. We injected both types from various directions, the dipole from the directions of highest and lowest GMF transparency, the point sources additionally from directions of nearby sources, such as Centaurus A [32].

For all injected anisotropies, we found a strong isotropisation below roughly $1 \mathrm{EV}$ to the percent level. At higher rigidities, the only significant anisotropy that remained was that of a dipole for all injected anisotropies. Point sources had amplitudes of around $10 \%$, as opposed to a few $\%$ for the injected dipoles.

For both the injected dipole and point source, a spectral modification was observed. For the injected dipole, this modification manifested in the form of a change in the spectral index across

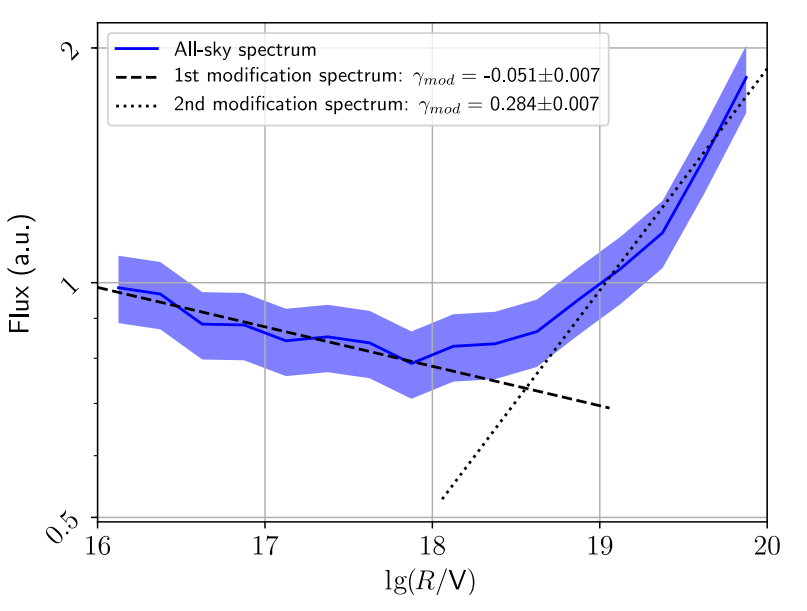

Figure 8: Rigidity spectrum of the lensed distribution from the direction of Centaurus A. the entire rigidity range, while for the point sources, a spectral break arose at rigidities of a few EV for some directions. The rigidity spectrum of the lensed distribution from the direction of Centaurus A is depicted in Fig. 8, where the spectral break is even "ankle"-like.

\section{Implications for the transition from Galactic to extragalactic cosmic rays}

We found that the propagation in the GMF of both GCRs and EGCRs in the energy range signifying the transition region may lead to a range of effects that modify the injected flux, composition, and arrival direction measured at Earth. We could plausibly tie these propagation effects to the change in propagation regimes with rigidity via the gyroradius, yielding a consistent picture of how the GMF modifies said observables.

The decreasing confinement of GCRs in the GP leads to a flux suppression towards higher rigidities, a "knee"-like feature that naturally arises without requiring a cut-off in the source spectrum. This in turn leads to an increased mean mass all the way up to "ankle" energies. As noted above, the heavier composition towards higher energies constitutes a propagation effect alone. Both a flux of EGCRs, as well as the introduction of a riditidy-dependent cut-off to GCR sources is required for a quantitative comparison with data, and both effects are expected to shift the maximum mean mass to lower energies. Beyond $0.1 \mathrm{EV}$, we additionally found a strong correlation in the arrival direction distribution with the source locations (i.e. direction of the GP), which also constrains the contribution of GCRs under the assumption of an even source distribution in the GP. 
EGCR observables are only affected in the case of an anisotropic injection. The GMF strongly isotropises the trajectories of EGCRs. This means a dipole can only survive above a rigidity of roughly $1 \mathrm{EV}$ with an amplitude of only the 1-10\%-level, depending on the nature of the dipole. More strikingly, the propagation in the GMF leads to a spectral modification, the nature and strength of which depend on the nature and direction of the anisotropy. An injected dipole leads to a smoother change with only a modification of the spectral index, while injection from a point source leads to spectral breaks in the rigidity range of a few EV. This is both roughly the energy of the "ankle" for protons and the rigidity at which the inflections occurred for the parameterisations of the previously discussed propagation effects. Especially noteworthy is that the injection from the direction of Centaurus A leads to a hardening of the spectrum, very similar in location and shape to that of the "ankle". This may partly tie the occurrence of the "ankle" to propagation effects in the GMF that arise from the change in propagation regimes with rigidity. It is also worth reiterating that we injected pure anisotropies, i.e. no isotropic component to the injected flux. For more realistic fluxes with both an isotropic and anisotropic component, the measured anisotropy and flux modification due to propagation in the GMF is expected to be weaker than found here.

With respect to the transition region, these findings suggest that higher-energy GCR sources in the GP are disfavoured judging from the effects that the GMF imposes on observables, particularly the arrival direction distribution. In this regard, alternative contributions such as re-acceleration in Galactic wind termination shocks (GWTSs) appear more promising [16, 17]. GWTSs can also be a source of lower-energy EGCRs. The difference is that GCRs from GWTSs diffuse back to the Galaxy after re-acceleration, whereas EGCRs from GWTSs are the ones that leak out of their host galaxies after re-acceleration. The most important finding for EGCRs pertaining to this work is the notion that anisotropic EGCR injections into the Galaxy (i.e. a large contribution from a small number of rather nearby sources) will result in a modification of observables after propagation through the GMF. Such modifications may manifest in "ankle"-like spectral breaks or smooth deformations of the spectra. Only significant dipolar large-scale anisotropies in the arrival direction are expected. However, to describe the measured spectral, composition and anisotropy data fully, additional assumptions about the injected spectrum need to be made, as changes from the GMF alone cannot account for them. In general, the sum of the GCR and EGCR contributions needs to be considered to account for the observed features in the transition region.

\section{Conclusion}

This work strongly suggests that the effects of CR propagation in the GMF on the spectrum, composition and arrival directions needs to be taken into account in the energy range signifying the transition from GCRs to EGCRs. We showed that this can be tied to the fact that the transition in propagation regimes from diffusive to ballistic also occurs in this energy range. While for GCRs, the main process that takes effect is the decreased confinement, EGCRs are affected more subtly via the directional shift in the GMF transparency.

Our work can be expanded upon most concretely by making more realistic assumptions about the source distribution and spectra. Particularly the effect of a discrete GCR source distribution may lead to additional or entirely new effects. Gauging the degree of expected anisotropy of EGCRs injected into the Galaxy is of special importance for understanding the effect that propagation in 
the GMF may have on CRs. Additionally, this work employs the ballistic propagation scheme of CRPropa where the equation of motion is solved. A complementary analysis using diffusive propagation solving the transport equation is particularly crucial to check the validity of our findings at low energies, but also to estimate at which energy diffusive and ballistic propagation must be assumed, respectively. Lastly, the effect of interactions, especially nuclear interactions during propagation, must be accounted for to get a full picture of the propagation effects in the Galaxy. These would mainly be of importance at lowest rigidities, possibly below the minimum rigidity employed for this work.

Additional remark: The results presented here are taken from the $\mathrm{PhD}$ thesis of Alex Kääpä which, at the time of publication of these proceedings, is to be yet to be completed. Further details to all analyses will be found therein.

Acknowledgments: The simulations were carried out on the Pleiades cluster at the University of Wuppertal, which was supported by the Deutsche Forschungsgemeinschaft (DFG). Further financial support by the German Ministry of Research and Education (Verbundforschung Astroteilchenphysik) is gratefully acknowledged.

\section{References}

[1] J. Becker Tjus and L. Merten, Phys. Rept. 872 (2020) 1 [2002 . 00964].

[2] W.D. Apel et al., [KASCADE Grande Coll.], Phys. Rev. Lett. 107 (2011) 171104 [1107. 5885].

[3] A. Aab et al., [Pierre Auger Coll.], Phys. Rev. Lett. 125 (2020) 121106 [2008. 06488].

[4] S. Thoudam, J.P. Rachen, A. van Vliet, A. Achterberg, S. Buitink, H. Falcke et al., Astron. Astrophys. 595 (2016) A33 [1605.03111].

[5] J. Becker Tjus, B. Eichmann, M. Kroll and N. Nierstenhöfer, Astropart. Phys. 81 (2016) 1 [1510.07801].

[6] R. Alves Batista et al., Front. Astron. Space Sci. 6 (2019) 23 [1903. 06714].

[7] K. Greisen, Phys. Rev. Lett. 16 (1966) 748.

[8] G.T. Zatsepin and V.A. Kuz'min, Soviet Journal of Experimental and Theoretical Physics Letters 4 (1966) 78.

[9] F.W. Stecker and M.H. Salamon, Astrophys. J. 512 (1999) 521 [astro-ph/9808110].

[10] V.S. Berezinsky and S.I. Grigor'eva, Astron. Astrophys. 199 (1988) 1.

[11] W.D. Apel et al., Phys. Rev. D 87 (2013) 081101 [1304.7114].

[12] J. Bellido, [Pierre Auger Coll.], PoS ICRC2017 (2018) 506.

[13] A. Aab et al., [Pierre Auger Coll.], JCAP 04 (2017) 038 [1612 . 07155], [Erratum: JCAP 03, E02 (2018)].

[14] A.M. Hillas, J. Phys. G 31 (2005) R95.

[15] P.L. Biermann et al., Adv. Space Res. 62 (2018) 2773 [1803. 10752].

[16] C. Bustard, E.G. Zweibel and C. Cotter, Astrophys. J. 835 (2017) 72 [1610. 06565].

[17] L. Merten, C. Bustard, E.G. Zweibel and J. Becker Tjus, Astrophys. J. 859 (2018) 63 [1803 . 08376].

[18] N. Globus, D. Allard and E. Parizot, Phys. Rev. D 92 (2015) 021302 [1505.01377].

[19] M. Unger, G.R. Farrar and L.A. Anchordoqui, Phys. Rev. D 92 (2015) 123001 [1505. 02153].

[20] M. Ahlers and P. Mertsch, Prog. Part. Nucl. Phys. 94 (2017) 184 [1612.01873].

[21] A. Aab et al., [Pierre Auger Coll.], Astrophys. J. 891 (2020) 142 [2002.06172]. 
[22] M.G. Aartsen et al., [IceCube Coll.], Astrophys. J. 826 (2016) 220 [1603.01227].

[23] A. Aab et al., [Pierre Auger Coll.], Science 357 (2017) 1266 [1709.07321].

[24] M. Erdmann, G. Müller, M. Urban and M. Wirtz, Astropart. Phys. 85 (2016) 54 [1607. 01645$].$

[25] G.R. Farrar and M.S. Sutherland, JCAP 05 (2019) 004 [1711.02730].

[26] B. Eichmann and T. Winchen, JCAP 04 (2020) 047 [2001. 01530].

[27] R. Jansson and G.R. Farrar, ApJ 757 (2012) 14 [1204 . 3662].

[28] R. Alves Batista, A. Dundovic, M. Erdmann, K.-H. Kampert, D. Kuempel, G. Müller et al., JCAP 05 (2016) 038 [1603.07142].

[29] T.K. Gaisser, R. Engel and E. Resconi, Cosmic Rays and Particle Physics: 2nd Edition, Cambridge University Press $(6,2016)$.

[30] K. Murase and M. Fukugita, Phys. Rev. D 99 (2019) 063012 [1806.04194].

[31] H.-P. Bretz, M. Erdmann, P. Schiffer, D. Walz and T. Winchen, Astropart. Phys. 54 (2014) 110 [1302. 3761].

[32] A. Aab et al., [Pierre Auger Coll.], Astrophys. J. Lett. 853 (2018) L29 [1801. 06160]. 